\title{
Formigas (Hymenoptera: Formicidae) Bioindicadoras de Degradação Ambiental em Poxoréu, Mato Grosso, Brasil
}

\author{
Wilian de Oliveira Rocha ${ }^{1}$, Alberto Dorval ${ }^{2}$, Otávio Peres Filho ${ }^{2}$, \\ Caroline dos Anjos Vaez ${ }^{2}$, Edilene Silva Ribeiro ${ }^{3}$
}

${ }^{1}$ Grupo de Produção Acadêmica de Ciências Agrárias, Biológicas e Engenharias, Curso de Engenharia Ambiental,
Centro Universitário de Várzea Grande - UNIVAG, Várzea Grande/MT, Brasil
2Programa de Pós-Graduação em Ciências Florestais e Ambientais, Departamento de Engenharia Florestal,
Universidade Federal de Mato Grosso - UFMT, Cuiabá/MT, Brasil
${ }^{3}$ Instituto Federal de Educação, Ciência e Tecnologia de Mato Grosso - IFMT, Campo Novo dos Parecis/MT, Brasil

\section{RESUMO}

Este trabalho teve por objetivo avaliar as espécies de formigas que ocorrem em áreas degradadas pela mineração de diamantes, definindo as espécies bioindicadoras desta degradação. O estudo realizado entre junho de 2010 e abril de 2011, bimestralmente, em Poxoréu - MT, amostrou quatro ambientes: A1 - área degradada pela mineração; A2 - área com vegetação de cerrado antropizado recentemente; A3 - vegetação antropizada há cerca de 30 anos; A4 - área de cerrado preservado. Em cada ambiente, foram instaladas 11 armadilhas pitfall, equidistantes 15 metros, para a coleta de formigas durante 24 horas. Os indivíduos coletados foram levados ao Laboratório de Proteção Florestal/FENF/UFMT e triados para posterior identificação. As espécies bioindicadoras foram indicadas por meio de análises faunísticas. As espécies Camponotus (Myrmaphaenus) sp.1 e Forelius brasiliensis podem ser consideradas bioindicadoras de degradação ambiental na área de garimpo de diamantes e antropização, enquanto Camponotus atriceps, Pachycondyla crassinoda e Paraponera clavata podem ser consideradas bioindicadoras de cerrado preservado.

Palavras-chave: indicadores biológicos, mineração, Cerrado.

\section{Ants (Hymenoptera: Formicidae) as Bioindicators of Environmental Degradation in Poxoréu, Mato Grosso, Brazil}

\begin{abstract}
This work aimed to evaluate the ant species occurring in an area degraded by diamond mining, defining the bioindicators of this degradation. The present study was conducted bimonthly in the municipality of Poxoréu, Mato Grosso state, between June 2010 and April 2011. Four environments were sampled: A1 - area degraded by mining, A2 - area of Cerrado vegetation with recent anthropic activities, A3 - area of Cerrado disturbed approximately 30 years ago, and A4 - area of preserved Cerrado. In each environment, 11 pitfall traps, equidistant 15 meters, were installed to collect ants for 24 hours. The individuals were taken to the Laboratory of Forest Protection/FENF/UFMT and screened for later identification. The bioindicator species were indicated by faunal analysis. The ant species Camponotus (Myrmaphaenus) sp.1 and Forelius brasiliensis can be considered bioindicators of environmental degradation in the areas of diamond mining and human disturbance, while the species Camponotus atriceps, Pachycondyla crassinoda and Paraponera clavata can be considered bioindicators in the preserved Cerrado area.
\end{abstract}

Keywords: biological indicators, mining, Cerrado. 


\section{INTRODUÇÃO}

As atividades humanas, como a mineração, contribuem decisivamente para a degradação do ambiente, modificando a área onde são implantadas, pois causam destruição e/ou alteração da vegetação natural e das características físico-químicas dos solos, interferindo nos cursos d'água, além de modificar o habitat da fauna e vários outros caracteres ambientais (Rocha, 2008).

A recuperação de áreas degradadas deve ser subsidiada em pesquisas científicas que garantam metodologias corretas e eficazes para se atingirem os objetivos desejados. Não é recomendável que se faça diretamente a execução de práticas de recuperação sem antes analisar metodicamente a área degradada. É fundamental, primeiramente, conhecer o nível de degradação e o seu potencial de regeneração, com o propósito de se obter sucesso na recuperação desses ambientes.

Uma das maneiras de detectar e monitorar os padrões de mudança na biodiversidade, provocados por ações antrópicas, é utilizar espécies - ou mesmo grupo de espécies - que atuam como bioindicadoras da degradação ambiental (Santos et al., 2006), pois a presença ou a ausência de uma população pode servir como parâmetro a ser avaliado (Ré, 2007).

$\mathrm{O}$ equilíbrio ambiental pode ser medido pela observação das características populacionais de grupos de organismos específicos, considerados bioindicadores do grau de alteração ou fragmentação de um local. Os mais importantes indicadores são os insetos, tanto por serem o grupo mais diverso em número de espécies como pela facilidade de amostragem. Então, a diversidade de insetos edáficos pode revelar o nível de qualidade ambiental a partir do qual podem ser determinadas intervenções a fim de manter, recuperar ou restaurar o equilíbrio do ambiente, atingindo, assim, a sustentabilidade ecológica dos ecossistemas (Wink et al., 2005).

Diversos grupos de insetos têm sido utilizados para este fim por apresentarem alta diversidade $e$ suscetibilidade às mudanças dos ambientes físico e biológico, entre os quais os Formicidae, da Ordem Hymenoptera (Santos et al., 2006).

As formigas se constituem em um dos grandes grupos de invertebrados nas regiões tropicais, pois apresentam alta riqueza de espécies e são ecologicamente importantes na serapilheira de espécies florestais tropicais (Agosti et al., 2000).

A riqueza e a diversidade de espécies de formigas tendem a aumentar de acordo com a complexidade dos ambientes, devido a uma maior disponibilidade de nichos presentes. Em razão da conexão entre os organismos e as características dos habitats, as formigas têm sido utilizadas como ferramentas no monitoramento ambiental de áreas perturbadas (Pereira et al., 2007), por apresentarem uma grande quantidade de características desejáveis, como facilidade de amostragem; alta abundância e ampla distribuição; importância no funcionamento dos ecossistemas, e ecologia e taxonomia relativamente bem conhecidas (Agosti et al., 2000).

Os formicídeos estão sendo utilizados em diversas áreas, como em minerações, na avaliação por contaminação de pesticidas, em distúrbios de habitats e na avaliação de impacto do desmatamento (Majer, 1983; Vasconcelos, 1999; Françoso \& Corrêa, 2007; Ré, 2007; Valentim et al., 2007; Valentim, 2010).

Dentre os diversos métodos de amostragem utilizados em levantamentos faunísticos de formigas, destacam-se: as coletas manuais; coletas com iscas; extratores de Winkler; agitação de folhagens, e as armadilhas do tipo Pitfall - consideradas eficientes, de baixo custo e fácil instalação, podendo permanecer no local por extensos períodos (Ré, 2007).

O grau de degradação ou recuperação de uma área pode ser definido através de estudos simples da fauna de formigas, detectando-se a presença ou as ausências de espécies raras e indicadoras de um estado de sucessão definido, através de estudos de similaridade ou dissimilaridade de populações em diferentes áreas, já que estas variam, conforme o estado de sucessão da vegetação (Kremen et al., 1993).

Diversas pesquisas, ao avaliarem o impacto ambiental das ações antrópicas sobre os ambientes naturais, utilizaram a análise faunística. Para realizar esta análise, os insetos coletados foram caracterizados através dos mais diversos índices faunísticos existentes (Fowler., 1991; Silveira Neto et al., 1995; Ré, 2007; Rocha, 2007; Bihn et al., 2008; Oliveira, 2009).

As estreitas relações das formigas com a vegetação demonstram que o tipo e a intensidade da degradação ou da alteração do ambiente podem produzir respostas 
diferenciadas, sendo algumas espécies beneficiadas e outras prejudicadas, diante dessas modificações ambientais (Oliveira, 2009).

Entre as pesquisas recentes, não existem informações destes bioindicadores para áreas degradadas pela garimpagem de diamantes no bioma Cerrado, especialmente utilizando formigas (Hymenoptera: Formicidae). Portanto, não há a possibilidade de avaliar com fidelidade este tipo de degradação, já que não existe conhecimento das espécies de formigas bioindicadoras para esta situação.

Neste estudo, objetivou-se avaliar qualitativa e quantitativamente as espécies de formigas (Hymenoptera) que ocorrem associadas às áreas de garimpo de diamantes em Poxoréu-MT, além de identificar as possíveis espécies com potencial de bioindicadores ambientais para áreas relacionadas a esta atividade econômica.

\section{MATERIAL E MÉTODOS}

\section{1. Área de estudo}

O estudo foi realizado na Fazenda Limeira (1544'50"S e $\left.54^{\circ} 26^{\prime} 09^{\prime \prime} \mathrm{W}\right)$, no município de Poxoréu, Estado de Mato Grosso, Brasil, onde predomina a ocorrência de áreas degradadas por atividade de garimpos (mineração) de diamantes, no bioma Cerrado. Em parte da fazenda, existem escavações de grandes proporções, com solo totalmente exposto e revolvido, encontrando também diversos amontoados de cascalhos e pedregulhos. Também, em área adjacente a esta, existe vegetação de cerrado antropicamente modificada, além de porções intactas, que conserva a biodiversidade característica da região.

A área amostral compreende a fitofisionomia Cerrado stricto sensu (ou sentido restrito). Esse tipo de vegetação apresenta árvores baixas, inclinadas e tortuosas, em que estão presentes arbustos e subarbustos espalhados em meio ao estrato herbáceo composto, predominantemente, por gramíneas (Ribeiro \& Walter, 1998).

O solo da região apresenta muitas variações, sendo os mais comuns, o Latossolo Vermelho-Amarelo e o Latossolo Vermelho-Escuro, com média a baixa fertilidade. A altitude média é de $450 \mathrm{~m}$ acima do nível do mar. O clima é Aw (Köppen), com temperatura média anual de $22^{\circ} \mathrm{C}$ e precipitação anual de $1.750 \mathrm{~mm}$ a $1.900 \mathrm{~mm}$, tendo intensidade máxima nos meses de dezembro, janeiro e fevereiro (Mato Grosso, 2009).

Nesta propriedade, predominam quatro ambientes distintos, de acordo com um gradiente de degradação ambiental promovido pelas atividades do garimpo de diamantes, sendo:

- Ambiente 1 (A1) - Área degradada pela mineração (garimpagem) de diamantes, com vegetação totalmente removida e solo exposto, apresentando alto nível de erosão;

- Ambiente 2 (A2) - Área adjacente ao Ambiente 1, com vegetação ainda estabelecida, porém foi antropizada pela instalação e vivência dos trabalhadores do garimpo (garimpeiros) há cerca de dez anos; encontra-se limitada pela estrada que secciona a área de estudo; - Ambiente 3 (A3) - Área adjacente ao Ambiente 2, sendo separada deste, por um lado, pela estrada que secciona a propriedade, e delimitada, de outro lado, pela área de cerrado inteiramente preservado. Apresenta, assim como o ambiente anterior, vegetação ainda estabelecida com vestígios da presença humana dos garimpos provenientes de 30 anos;

- Ambiente 4 (A4) - Área adjacente ao Ambiente 3, com vegetação preservada, solo inteiramente coberto por serrapilheira e pelo dossel típico do cerrado sensu stricto, sem sinais de atividades antrópicas.

\subsection{Coleta de dados}

Em cada ambiente, foi instalado um transecto de 150 metros de comprimento, no qual foram distribuídas 11 armadilhas do tipo pitfall, com uma distância de 15 metros entre cada armadilha.

As armadilhas, modelo pitfall, foram confeccionadas com potes plásticos transparentes de $300 \mathrm{~mL}$, com $15 \mathrm{~cm}$ de altura e $10 \mathrm{~cm}$ de diâmetro. Estas foram enterradas com suas bordas no nível da superfície do solo, sendo preenchidas com solução conservante contendo água, detergente neutro e sal, a um terço da capacidade do recipiente (Aquino et al., 2006). Após o período de 24 horas, as armadilhas foram retiradas e o conteúdo coletado foi devidamente individualizado por armadilha, armazenado e etiquetado com as características do local. Foram realizadas coletas bimestrais, no período de junho de 2010 a abril de 2011, totalizando seis coletas e 264 amostras.

Os espécimes coletados foram transportados ao Laboratório de Proteção Florestal (LAPROFLOR), da Faculdade de Engenharia Florestal (FENF), da 
Universidade Federal de Mato Grosso (UFMT), para triagem e contagem das formigas. Os espécimes morfologicamente diferentes foram enviados ao Dr. Jacques Hubert C. Delabie - Laboratório de Mirmecologia do Centro de Pesquisas do Cacau (CEPEC), da Comissão Executiva do Plano da Lavoura Cacaueira (CEPLAC), Ilhéus, Bahia - para a devida identificação taxonômica em nível específico.

\subsection{Análise dos dados}

Após a identificação e a quantificação dos indivíduos de cada espécie, realizaram-se estudos faunísticos de constância, dominância, abundância e frequência através do programa ANAFAU (Moraes et al., 2003), que calcula estes índices através dos métodos propostos por Laroca e Mielke (1975) e Sakagami e Laroca (1967), para, assim, apontar possíveis espécies bioindicadoras da degradação relacionada à mineração de diamantes nesta localidade.

\section{RESULTADOS E DISCUSSÃO}

\subsection{Análises quantitativa e qualitativa}

Nos quatro ambientes, foram coletados 10.521 indivíduos, sendo 2.665 (25,30\%) espécimes em A1; 3.625 (34,42\%) em A2; 1.934(18,36\%) em A3, e 2.307 (21,90\%) em A4.

Foram coletadas 15 espécies nos quatro ambientes amostrados, distribuídas nas subfamílias Dolichoderinae, Ectatomminae, Formicinae, Myrmicinae e Ponerinae (Tabela 1).

Nos quatro ambientes amostrados, observou-se a ocorrência de sete espécies comuns a todos os ambientes. Esse fato é devido ao hábito alimentar igual de seis dessas espécies que, sendo onívoras, adaptam-se com facilidade em diferentes ambientes. Note-se que a espécie distinta - Ectatomma opaciventre - é considerada predadora de pequenos invertebrados,

Tabela 1. Subfamília, espécies, hábito alimentar e quantidade de indivíduos coletados com armadilhas pitfall nos quatro ambientes em Poxoréu, Mato Grosso, Brasil - junho de 2010 a abril de 2011.

Table 1. Sub-family, species, feeding habit and number of individuals collected using pitfall traps in four environments in Poxoréu, Mato Grosso, Brazil. June 2010 to April 2011.

\begin{tabular}{|c|c|c|c|c|c|c|c|}
\hline \multirow{2}{*}{ Subfamílias/ Espécies } & \multirow{2}{*}{ H.A. } & A1 & A2 & A3 & A4 & \multirow{2}{*}{ Total } & \multirow[t]{2}{*}{$\%$} \\
\hline & & & I & $\mathbf{I}$ & I & & \\
\hline \multicolumn{8}{|l|}{ Dolichoderinae } \\
\hline Forelius brasiliensis (Forel, 1908) & $\mathrm{O}$ & 152 & 14 & 123 & - & 289 & 2,75 \\
\hline \multicolumn{8}{|l|}{ Ectatomminae } \\
\hline Ectatomma edentatum Roger, 1863 & $\operatorname{Pr}$ & - & 2 & 8 & - & 10 & 0,10 \\
\hline Ectatomma opaciventre Roger, 1861 & $\operatorname{Pr}$ & 53 & 49 & 40 & 14 & 156 & 1,48 \\
\hline \multicolumn{8}{|l|}{ Formicinae } \\
\hline Brachymyrmex patagonicus Mayr, 1868 & $\mathrm{O}$ & 1.771 & 1.759 & 483 & 1.402 & 5.415 & 51,4 \\
\hline Brachymyrmex pilipes Mayr, 1887 & $\mathrm{O}$ & 145 & 1.017 & 8 & 70 & 1.240 & 11,8 \\
\hline Camponotus (Myrmaphaenus) sp. & $\mathrm{O}$ & 374 & 135 & 163 & 42 & 714 & 6,8 \\
\hline Camponotus atriceps (Smith, 1858) & $\mathrm{O}$ & - & - & 74 & 289 & 363 & 3,45 \\
\hline Camponotus crassus Mayr, 1862 & $\mathrm{O}$ & 1 & 47 & 325 & 150 & 523 & 4,97 \\
\hline Camponotus melanoticus Emery, 1894 & $\mathrm{O}$ & - & 246 & 397 & 121 & 764 & 7,26 \\
\hline Camponotus vittatus Forel, 1904 & $\mathrm{O}$ & 20 & 14 & 2 & 6 & 42 & 0,4 \\
\hline \multicolumn{8}{|l|}{ Myrmicinae } \\
\hline Cephalotes pusillus (Klug, 1824) & $\mathrm{O}$ & 10 & - & 3 & 1 & 14 & 0,13 \\
\hline Solenopsis substituta (Santischi) & $\mathrm{O}$ & 129 & 271 & 216 & 70 & 686 & 6,52 \\
\hline Trachymyrmex sp.1 & Cf & - & 9 & 46 & - & 55 & 0,52 \\
\hline \multicolumn{8}{|l|}{ Ponerinae } \\
\hline $\begin{array}{l}\text { Pachycondyla crassinoda } \\
\text { (Latreille,1802) }\end{array}$ & $\mathrm{O}$ & - & 52 & 22 & 86 & 160 & 1,52 \\
\hline Paraponera clavata (Fabricius, 1775) & $\mathrm{O}$ & - & 10 & 24 & 56 & 90 & 0,86 \\
\hline Total & & 2.655 & 3.625 & 1.934 & 2.307 & 10.521 & 100 \\
\hline
\end{tabular}

H.A.: Hábito Alimentar, sendo O - Onívora, $\mathrm{Pr}$ - Predadora, Cf - Cultivadora de fungos; A1: Ambiente 1; A2: Ambiente 2; A3: Ambiente 3; A4: Ambiente 4; I : Indivíduos; \%: Porcentagem de indivíduos. 
característica necessária ao equilíbrio da diversidade ambiental. Esta ocorrência demonstra a proporção desses dois níveis tróficos dentro da cadeia alimentar nessa região (Tabela 1 ).

A subfamília Dolichoderinae possuiu uma espécie amostrada, Forelius brasiliensis, cujos indivíduos foram encontrados nos Ambientes A1, A2 e A3. Das duas espécies da subfamília Ectatomminae, somente Ectatomma opaciventre foi coletada nos quatro ambientes, enquanto Ectatomma edentatum teve ocorrência restrita aos ambientes A2 e A3 (Tabela 1).

Na subfamília Myrmicinae, Solenopsis substituta foi comum a todos os ambientes, confirmando seus hábitos generalistas; Cephalotes pusillus foi coletada nos ambientes A1, A3 e A4, provavelmente em meio à sua onivoria; a espécie Trachymyrmex sp.1 foi amostrada somente nos ambientes A2 e A3. As espécies de Pachycondyla crassinoda e Paraponera clavata (Ponerinae) ocorreram nos ambientes 2, 3 e 4 (Tabela 1).

As espécies do gênero Trachymyrmex são típicas de ambientes florestados e dependem de condições específicas de sombreamento, umidade e temperatura para sobreviverem, cultivando fungos para sua alimentação (Ré, 2007). Assim, também, $P$. crassinoda e $P$. clavata possuem uma necessidade ecológica de colonizar ambientes arborizados com boa produção de serapilheira, uma característica das Ponerinae. Dessa forma, essas espécies foram coletadas somente nos ambientes cuja vegetação nativa encontra-se estabelecida, ocorrendo em maior quantidade de indivíduos no ambiente A4, onde a vegetação do cerrado encontra-se preservada e sem qualquer tipo de perturbação.

A subfamília Formicinae foi a mais diversificada em quantidade de espécies, sendo Brachymyrmex patagonicus, Brachymyrmex pilipes, Camponotus (Myrmaphaenus) sp. 1, Camponotus crassus e Camponotus vittatus coletadas nos quatro ambientes estudados, enquanto Camponotus melanoticus ocorreu nos ambientes A2, A3 e A4, e Camponotus atriceps, somente em A3 e A4 (Tabela 1).

Formicinae também foi a subfamília mais representativa em quantidade de indivíduos coletados, independentemente do ambiente estudado. $\mathrm{Na}$ análise conjunta de todos os ambientes, as espécies B. patagonicus, com 5.415 (51,47\%) indivíduos; B. pilipes, com 1.240 (11,79\%); C. melanoticus, com 764 (7,26\%), e C. (Myrmaphaenus) sp., com 714 (6,79\%), evidenciam essa representatividade (Tabela 1 ).

As espécies de Brachymyrmex são forrageiras dominantes de serapilheira e onívoras (Delabie et al., 2000; Ré, 2007), o que possibilitou o estabelecimento de colônias em todos os ambientes estudados.

As espécies do gênero Camponotus (Formicinae) também são onívoras e nidificam em galhos de árvores; são consideradas pouco agressivas, sendo amplamente encontradas no bioma de cerrado e ambientes urbanos (Fowler, 1991; Bolton, 2003; Rocha \& Solferini, 2008), o que explica esta ocorrência em todos os ambientes amostrados, desde os antropizados até o cerrado preservado, além de apresentar a maior quantidade de espécies quando comparado aos demais gêneros.

\subsection{Análise faunística}

Observou-se, através do método de Laroca e Mielke (1975), que ocorreram no Ambiente 1: uma espécie super dominante, sete espécies dominantes e uma não dominante. Pelo método de Sakagami e Laroca (1967), verificou-se uma espécie superdominante, quatro dominantes e quatro não dominantes. Quanto à abundância, uma espécie foi superabundante, uma muito abundante, quatro espécies comuns, uma dispersa e duas raras. Com relação à frequência, uma espécie foi superfrequente, uma muito frequente, quatro frequentes e três pouco frequentes. Quanto à constância, oito espécies foram constantes e uma acidental (Tabela 2).

A espécie $B$. patagonicus foi mais representativa no ambiente A1, ocorrendo como superdominante, superabundante, superfrequente e constante, enquanto C. (Myrmaphaenus) sp.1 ocorreu como dominante, muito abundante, muito frequente e constante. Em contrapartida, a menor representatividade foi demonstrada por C. crassus em A1, sendo considerada não dominante, rara, pouco frequente e acidental (Tabela 2).

Pelo método de Laroca e Mielke (1975), ocorreram duas espécies superdominantes, dez dominantes e uma não dominante em A2, enquanto que, pelo método de Sakagami e Laroca (1967), duas espécies foram super dominantes, três dominantes e oito não dominantes. Com relação à abundância, duas espécies foram superabundantes, duas muito abundantes, uma abundante, três comuns e cinco dispersas. Com relação à frequência, ocorreram duas espécies superfrequentes, 
Tabela 2. Índices faunísticos das espécies coletadas no Ambiente 1 (A1) em Poxoréu, Mato Grosso, Brasil - junho de 2010 a abril de 2011.

Table 2. Faunistic indices of species collected in Environment 1 (A1) in Poxoréu, Mato Grosso, Brazil. June 2010 to April 2011.

\begin{tabular}{|c|c|c|c|c|c|c|}
\hline \multirow{3}{*}{ Gênero/Espécie } & \multirow{3}{*}{ Quantidade de indivíduos } & \multicolumn{5}{|c|}{ Índices Faunísticos } \\
\hline & & \multicolumn{2}{|c|}{ D } & \multirow{2}{*}{ A } & \multirow{2}{*}{$\mathbf{F}$} & \multirow{2}{*}{ C } \\
\hline & & 1 & 2 & & & \\
\hline Camponotus (Myrmaphaenus) sp. 1 & 374 & $\mathrm{~d}$ & $\mathrm{~d}$ & $\mathrm{ma}$ & $\mathrm{mf}$ & $\mathrm{w}$ \\
\hline Brachymyrmex pilipes & 145 & $\mathrm{~d}$ & $\mathrm{~d}$ & c & $\mathrm{f}$ & w \\
\hline Brachymyrmex patagonicus & 1.771 & sd & sd & sa & sf & $\mathrm{w}$ \\
\hline Ectatomma opaciventre & 53 & $\mathrm{~d}$ & nd & c & $\mathrm{f}$ & w \\
\hline Forelius brasiliensis & 152 & $\mathrm{~d}$ & d & c & $\mathrm{f}$ & $\mathrm{w}$ \\
\hline Cephalotes pusillus & 10 & $\mathrm{~d}$ & nd & $\mathrm{r}$ & $\mathrm{pf}$ & w \\
\hline Camponotus vittatus & 20 & d & nd & d & $\mathrm{pf}$ & $\mathrm{w}$ \\
\hline Solenopsis substituta & 129 & $\mathrm{~d}$ & d & c & $\mathrm{f}$ & w \\
\hline Camponotus crassus & 1 & nd & nd & $\mathrm{r}$ & $\mathrm{pf}$ & $\mathrm{z}$ \\
\hline
\end{tabular}

Programa ANAFAU: (1) Método de Laroca e Mielke (1975); (2) método de Sakagami e Laroca (1967). D: Dominância - (sd) superdominante; (d) dominante; (nd) não dominante. A: Abundância - (sa) superabundante; (ma) muito abundante; (a) abundante; (c) comum; (d) dispersa; (r) rara. F: Frequência: (sf) superfrequente; (mf) muito frequente; (f) frequente; (pf) pouco frequente. C: Constância - (w) constante; (y) acessória; (z) acidental.

três muito frequentes, três frequentes e cinco espécies pouco frequentes. Quanto à constância, ocorreram 11 classificadas como constantes e duas acessórias (Tabela 3).

Neste ambiente, $B$. patagonicus e $B$. pilipes foram as espécies mais representativas em quantidade de indivíduos coletados. Na análise faunística, ocorreram como super dominantes, super abundantes, super frequentes e constantes, enquanto $S$. substituta e C. melanoticus ocorreram como dominantes, muito abundantes, muito frequentes e constantes. E inversamente, a espécie menos representativa em A2 foi E. edentatum (Tabela 3).

Destacam-se como dominantes e muito abundantes, e muito frequentes, as espécies B. patagonicus, C. melanoticus e C. crassus com, respectivamente, 483, 397 e 395 indivíduos. E vale ressaltar C. vittatus como uma espécie rara e acidental neste ambiente (Tabela 4).

Ao analisar o ambiente A4, pelo método de Laroca e Mielke (1975) ocorreu uma espécie super dominante, dez dominantes e uma não dominante e pelo método de Sakagami e Laroca (1967) ocorreu uma espécie super dominante, quatro dominantes e sete não dominantes. Quanto a abundância, uma espécie foi super abundante, duas muito abundantes, seis comuns e três raras. Com relação à frequência, uma espécie foi superfrequente, duas muito frequentes, seis frequentes e três pouco frequentes e em relação à constância, dez espécies foram constantes, uma acessória e uma acidental. Assim, B. patagonicus foi a espécie mais representativa, ocorrendo como super dominante, super abundante, superfrequente e constante (Tabela 5).

Já C. pusillus e C. vittatus foram consideradas raras quanto à frequência e, respectivamente, acidental e acessória para a constância, estendendo esses baixos níveis para os demais índices de acordo com a amostragem em A4 (Tabela 5).

Ao verificar as análises de fauna obtidas para cada ambiente, é notável a utilização de dois índices de dominância pelo ANAFAU (Moraes et al, 2003), que analisados em conjunto para este tipo de avaliação pode determinar com maior eficácia a bioindicação de determinada espécie em uma análise conjunta com os demais índices fornecidos. Já que um organismo dominante em uma comunidade é aquele que possui capacidade de sofrer o impacto do meio e responder de forma saudável ou equilibrada, mantendo-se no local e alterando o próprio ambiente a sua volta, essa espécie pode interferir nas comunidades de outros organismos e, até mesmo, proporcionando o desaparecimento ou não destes (Silveira Neto et al., 1976).

As espécies consideradas com baixos índices como não dominantes, dispersas ou raras, pouco frequentes e acidentais, também são de importante interesse para a avaliação de um ambiente, mesmo que estes níveis 
Tabela 3. Indices faunísticos das espécies de formigas no Ambiente 2 (A2) em Poxoréu, Mato Grosso, Brasil - junho de 2010 a abril de 2011.

Table 3. Faunistic indices of species collected in Environment 2 (A2) in Poxoréu, Mato Grosso, Brazil. June 2010 to April 2011.

\begin{tabular}{|c|c|c|c|c|c|c|}
\hline \multirow{3}{*}{ Gênero/Espécie } & \multirow{3}{*}{$\begin{array}{l}\text { Quantidade } \\
\text { de indivíduos }\end{array}$} & \multicolumn{5}{|c|}{ Índices Faunísticos } \\
\hline & & \multicolumn{2}{|c|}{$\mathbf{D}$} & \multirow{2}{*}{ A } & \multirow{2}{*}{$\mathbf{F}$} & \multirow{2}{*}{ C } \\
\hline & & 1 & 2 & & & \\
\hline Camponotus (Myrmaphaenus) sp. 1 & 135 & $\mathrm{~d}$ & $\mathrm{~d}$ & a & $\mathrm{mf}$ & $\mathrm{w}$ \\
\hline Brachymyrmex pilipes & 1.017 & sd & sd & sa & sf & w \\
\hline Brachymyrmex patagonicus & 1.759 & sd & sd & sa & sf & $\mathrm{w}$ \\
\hline Ectatomma opaciventre & 49 & $\mathrm{~d}$ & nd & c & $\mathrm{f}$ & w \\
\hline Forelius brasiliensis & 14 & $\mathrm{~d}$ & nd & d & $\mathrm{pf}$ & $\mathrm{w}$ \\
\hline Camponotus vittatus & 14 & $\mathrm{~d}$ & nd & d & $\mathrm{pf}$ & $\mathrm{y}$ \\
\hline Solenopsis substituta & 271 & $\mathrm{~d}$ & $\mathrm{~d}$ & $\mathrm{ma}$ & $\mathrm{mf}$ & $\mathrm{w}$ \\
\hline Paclycondyla crassinoda & 52 & $\mathrm{~d}$ & nd & c & $\mathrm{f}$ & w \\
\hline Camponotus crassus & 47 & $\mathrm{~d}$ & nd & c & $\mathrm{f}$ & $\mathrm{w}$ \\
\hline Camponotus melanoticus & 246 & d & d & $\mathrm{ma}$ & $\mathrm{mf}$ & $\mathrm{w}$ \\
\hline Paraponera clavata & 10 & $\mathrm{~d}$ & nd & d & $\mathrm{pf}$ & $\mathrm{w}$ \\
\hline Trachymyrmex sp. 1 & 9 & d & nd & d & $\mathrm{pf}$ & $\mathrm{w}$ \\
\hline Ectatomma edentatum & 2 & nd & nd & d & $\mathrm{pf}$ & $\mathrm{y}$ \\
\hline
\end{tabular}

Programa ANAFAU: (1) Método de Laroca e Mielke (1975); (2) método de Sakagami e Laroca (1967). D: Dominância - (sd) super dominante; (d) dominante; (nd) não dominante. A: Abundância - (sa) superabundante; (ma) muito abundante; (a) abundante; (c) comum; (d) dispersa; (r) rara. F: Frequência: (sf) superfrequente; (mf) muito frequente; (f) frequente; (pf) pouco frequente. C: Constância - (w) constante; (y) acessória; (z) acidental.

Tabela 4. Índices faunísticos das espécies de formigas no Ambiente 3 (A3) em Poxoréu, Mato Grosso, Brasil. Junho de 2010 a abril de 2011.

Table 4. Faunistic indices of species collected in Environment 3 (A3) in Poxoréu, Mato Grosso, Brazil. June 2010 to April 2011.

\begin{tabular}{|c|c|c|c|c|c|c|}
\hline \multirow{3}{*}{ Gênero/Espécie } & \multirow{3}{*}{$\begin{array}{l}\text { Número de indi- } \\
\text { víduos }\end{array}$} & \multicolumn{5}{|c|}{ Índices Faunísticos } \\
\hline & & \multicolumn{2}{|c|}{ D } & \multirow{2}{*}{$\mathbf{A}$} & \multirow{2}{*}{$\mathbf{F}$} & \multirow{2}{*}{ C } \\
\hline & & 1 & 2 & & & \\
\hline Camponotus (Myrmaphaenus) sp. 1 & 163 & $\mathrm{~d}$ & $\mathrm{~d}$ & c & $\mathrm{f}$ & $\mathrm{w}$ \\
\hline Brachymyrmex pilipes & 8 & d & nd & $\mathrm{r}$ & $\mathrm{pf}$ & $\mathrm{w}$ \\
\hline Brachymyrmex patagonicus & 483 & d & $\mathrm{d}$ & $\mathrm{ma}$ & $\mathrm{mf}$ & $\mathrm{w}$ \\
\hline Ectatomma opaciventre & 40 & d & nd & c & $\mathrm{f}$ & $\mathrm{w}$ \\
\hline Forelius brasiliensis & 123 & d & nd & c & $\mathrm{f}$ & $\mathrm{w}$ \\
\hline Cephalotes pusillus & 3 & nd & nd & $\mathrm{r}$ & $\mathrm{pf}$ & w \\
\hline Camponotus vittatus & 2 & nd & nd & $\mathrm{r}$ & pf & z \\
\hline Solenopsis substituta & 216 & $\mathrm{~d}$ & $\mathrm{~d}$ & a & $\mathrm{mf}$ & $\mathrm{w}$ \\
\hline Pachycondyla crassinoda & 22 & d & nd & $\mathrm{r}$ & $\mathrm{pf}$ & $\mathrm{w}$ \\
\hline Camponotus crassus & 325 & d & d & $\mathrm{ma}$ & $\mathrm{mf}$ & w \\
\hline Camponotus melanoticus & 397 & d & $\mathrm{d}$ & $\mathrm{ma}$ & $\mathrm{mf}$ & $\mathrm{w}$ \\
\hline Paraponera clavata & 24 & d & nd & $\mathrm{r}$ & $\mathrm{pf}$ & $\mathrm{w}$ \\
\hline Trachymyrmex sp.1 & 46 & d & nd & d & $\mathrm{pf}$ & $\mathrm{w}$ \\
\hline Ectatomma edentatum & 8 & d & nd & $\mathrm{r}$ & $\mathrm{pf}$ & $\mathrm{w}$ \\
\hline Camponotus atriceps & 74 & d & nd & $\mathrm{c}$ & $\mathrm{f}$ & $\mathrm{w}$ \\
\hline
\end{tabular}

Programa ANAFAU: (1) Método de Laroca e Mielke (1975); (2) método de Sakagami e Laroca (1967). D: Dominância - (sd) super dominante; (d) dominante; (nd) não dominante. A: Abundância - (sa) super abundante; (ma) muito abundante; (a) abundante; (c) comum; (d) dispersa; (r) rara. F: Frequência: (sf) super frequente; (mf) muito frequente; (f) frequente; (pf) pouco frequente. C: Constância - (w) constante; (y) acessória; (z) acidental. 
Tabela 5. Índices faunísticos das espécies coletadas no Ambiente 4 (A4) em Poxoréu, Mato Grosso, Brasil. Junho de 2010 a abril de 2011.

Table 5. Faunistic indices of species collected in Environment 4 (A4) in Poxoréu, Mato Grosso, Brazil. June 2010 to April 2011.

\begin{tabular}{|c|c|c|c|c|c|c|}
\hline \multirow{3}{*}{ Gênero/Espécie } & \multirow{3}{*}{$\begin{array}{l}\text { Quantidade } \\
\text { de indi- } \\
\text { víduos }\end{array}$} & \multicolumn{5}{|c|}{ Índices Faunísticos } \\
\hline & & \multicolumn{2}{|c|}{ D } & \multirow{2}{*}{ A } & \multirow{2}{*}{$\mathbf{F}$} & \multirow{2}{*}{ C } \\
\hline & & 1 & 2 & & & \\
\hline Camponotus (Myrmaphaenus) sp. 1 & 42 & $\mathrm{~d}$ & nd & c & $\mathrm{f}$ & $\mathrm{w}$ \\
\hline Brachymyrmex pilipes & 70 & $\mathrm{~d}$ & nd & c & $\mathrm{f}$ & $\mathrm{w}$ \\
\hline Brachymyrmex patagonicus & 1.402 & sd & sd & sa & sf & $\mathrm{w}$ \\
\hline Ectatomma opaciventre & 14 & $\mathrm{~d}$ & nd & $\mathrm{r}$ & $\mathrm{pf}$ & $\mathrm{w}$ \\
\hline Cephalotes pusillus & 1 & nd & nd & $\mathrm{r}$ & $\mathrm{pf}$ & $\mathrm{z}$ \\
\hline Camponotus vittatus & 6 & $\mathrm{~d}$ & nd & $\mathrm{r}$ & $\mathrm{pf}$ & $\mathrm{y}$ \\
\hline Solenopsis substituta & 70 & $\mathrm{~d}$ & nd & c & $\mathrm{f}$ & $\mathrm{w}$ \\
\hline Pachycondyla crassinoda & 86 & $\mathrm{~d}$ & $\mathrm{~d}$ & c & $\mathrm{f}$ & w \\
\hline Camponotus crassus & 150 & $\mathrm{~d}$ & $\mathrm{~d}$ & $\mathrm{ma}$ & $\mathrm{mf}$ & w \\
\hline Camponotus melanoticus & 121 & $\mathrm{~d}$ & $\mathrm{~d}$ & c & $\mathrm{f}$ & w \\
\hline Paraponera clavata & 56 & $\mathrm{~d}$ & nd & c & $\mathrm{f}$ & $\mathrm{w}$ \\
\hline Camponotus atriceps & 289 & d & $\mathrm{d}$ & $\mathrm{ma}$ & $\mathrm{mf}$ & $\mathrm{w}$ \\
\hline
\end{tabular}

Programa ANAFAU:(1) Método de Laroca e Mielke (1975); (2) método de Sakagami e Laroca (1967). D: Dominância - (sd) super dominante; (d) dominante; (nd) não dominante. A: Abundância - (sa) super abundante; (ma) muito abundante;(a) abundante; (c) comum; (d) dispersa; (r) rara. F: Frequência: (sf) super frequente; (mf) muito frequente; (f) frequente; (pf) pouco frequente. C: Constância - (w) constante; (y) acessória; $(\mathrm{z})$ acidental.

considerados baixos não sejam utilizados para inferir espécies bioindicadoras. A amostragem de espécies consideradas raras, porém ainda constantes, como C. pusillus em A3, demonstra que o ambiente pode vir a possuir determinada capacidade de recursos vitais para estas espécies, onde no momento essa disponibilidade permanece reduzida ao mínimo necessário para que estas espécies habitem de modo a não serem extintas na área, aguardando as devidas condições ambientais e interespecíficas para que suas colônias elevem seus níveis faunísticos. E pode ser que, o método de amostragem adotado não seja eficiente para coletar as espécies indicadas com baixos parâmetros faunísticos por esta análise, sendo necessários outros estudos com técnicas e tempo de coletas diferenciados para comprovar estes índices considerados inferiores.

As análises faunísticas obtidas para cada ambiente relataram que B. patagonicus ocorreu com os melhores índices em todos os ambientes estudados, demonstrando alta adaptabilidade, talvez devido ao seu hábito alimentar onívoro e o forrageio bem distribuído na serapilheira. Assim, mesmo tendo esse alto nível para todos os índices de fauna calculados e seguindo as literaturas citadas, seria errôneo determiná-la como espécie bioindicadora de degradação ou preservação, já que foi representativa em todos os ambientes, independente destes serem degradados/antropizados ou preservados. Então, é correto inferir apenas que essa espécie é a mais adaptada a todos os ambientes estudados, com capacidade generalista para utilizar os recursos de diferentes nichos nesta região.

Portanto, há a necessidade de que a análise faunística seja interpretada junto à análise quantitativa, levando em consideração as características que limitam os ambientes deste estudo, degradados ou não, e relacionando às características conhecidas de cada espécie.

Ao levar em consideração os resultados dos índices faunísticos aliados à análise quantitativa, percebe que C. (Myrmaphaenus) sp.1 apresenta potencial como bioindicadora para a área degradada pela garimpagem na região, por ser considerada dominante, muito abundante/abundante, muito frequente e constante tanto em A1 quanto em A2. Estes índices tenderam a diminuir conforme se avaliou essa espécie nos demais ambientes mais preservados, seguindo um decréscimo semelhante na análise quantitativa.

A espécie F. brasiliensis também pode ser indicada como bioindicadora potencial para os ambientes que já 
sofreram determinada degradação antrópica, pois foi amostrada na área minerada e nos ambientes adjacentes, tanto desta (A2) quanto do cerrado preservado (A3), locais que mesmo apresentando a vegetação habitual deste bioma já sofreram com a antropização provocada pelo assentamento dos garimpeiros. Ainda, há a ausência total dessa espécie no ambiente de cerrado preservado que, não foi antropizado. E assim, equivalente à análise em quantidade dos indivíduos amostrados dessa espécie.

Os índices faunísticos obtidos para F. brasiliensis comprovam a sua indicação como bioindicadora de ambientes antropizados, pois ocorreu como dominante, abundante, muito frequente e constante na área garimpada. O trabalho de Cuezzo (2000) mostrou que F. brasiliensis ocorre em parte da região sul da América do Sul estendendo-se até o Paraguai, porém não indicou a ocorrência em Mato Grosso. A presença dessa espécie demonstra que sua distribuição está se ampliando, muito provavelmente devido aos meios antrópicos de locomoção, o que pode explicar o estabelecimento da espécie nesta antiga área de garimpo. Portanto, são necessários maiores estudos para determinar a real distribuição desta espécie em Mato Grosso.

Dentre os ambientes e espécies estudados, C. atriceps apresentou potencial como bioindicadora de ambiente com vegetação inalterada, pois foi somente amostrada nos ambientes A3 e A4, ocorrendo como dominante, muito abundante, muito frequente e constante em A4, aliado à sua quantidade de indivíduos que aumentou conforme a conservação do ambiente. Não foi constatada sua presença em A1 e A2, considerado que são os ambientes com degradação mais intensa.

Esta ocorrência restrita de C. atriceps nos ambientes A3 e A4, pode estar relacionada à característica desta espécie ser diretamente afetada pela estrutura da vegetação devido ao seu hábito de forragear na base de árvores e arbustos (Bestelmeyer \& Schooley, 1999; Oliveira, 2009), já que esses ambientes apresentam uma estrutura de vegetação menos degradada quando comparados com o estado de conservação da vegetação dos demais ambientes.

As espécies de $P$. crassinoda e $P$. clavata confirmam em A4, o seu potencial como bioindicadoras de ambientes preservados e ecologicamente mais estabilizados (Oliveira, 2009), já que ocorrem dominantes, frequentes e constantes. Porém, quanto à abundância, estas espécies ocorreram como comum neste ambiente, fato que pode ser explicado pelo seu hábito alimentar voltado à predação e, portanto sua densidade populacional é totalmente dependente da disponibilidade de presas em potencial, comprovando seu nível trófico na cadeia alimentar nesse ambiente.

E ainda, $P$. crassinoda e $P$. clavata, popularmente conhecidas como tocandiras, além de predadoras de pequenos invertebrados, são coletoras de néctar floral e extraflorais em alguns casos, demonstrando uma evolução ecológica ao aumentar seu hábito alimentar (Ré, 2007; Pacheco et al., 2005). Já foram observadas espécies do gênero Pachycondyla transportando sementes de floresta primária para uma área de pastagem (Oliveira, 2009) e P. clavata transportando frutos de lixeira (Curatella americana L. - Dilleniaceae) para os ninhos (Pacheco et al., 2005), sendo dois gêneros com possível importância ecológica para a sucessão florestal, podendo contribuir para a regeneração de ambientes degradados.

\section{CONCLUSÕES}

- Camponotus (Myrmaphaenus) sp.1 pode ser considerada bioindicadora de degradação ambiental pelo garimpo de diamantes desta região;

- Forelius brasiliensis pode ser considerada espécie bioindicadora de alteração ambiental antrópica nesta região;

- Camponotus atriceps pode ser considerada espécie bioindicadora de ambiente com vegetação de cerrado inalterado nesta região;

- Pachycondyla crassinoda e Paraponera clavata podem ser bioindicadoras de ambientes preservados e estabilizados ecologicamente;

- Brachymyrmex patagonicus é a espécie mais adaptada a todos os ambientes estudados, independente do nível de degradação;

\section{AGRADECIMENTOS}

Ao Dr. Jacques Hubert C. Delabie e equipe do Laboratório de Mirmecologia do Centro de Pesquisas do Cacau (CEPEC) da Comissão Executiva do Plano da Lavoura Cacaueira (CEPLAC), Ilhéus, Bahia, pela identificação das espécies coletadas. Ao Sr. Manoel Lauro da Silva, técnico do Laboratório de Proteção Florestal (LAPROFLOR) da Faculdade de Engenharia Florestal (FENF) da Universidade Federal de Mato 
Grosso (UFMT), pela acuidade com o material triado e conselhos para as coletas de campo. Ao Sr. Leônidas dos Santos por dispor sua propriedade para esta pesquisa.

\section{STATUS DA SUBMISSÃO}

Recebido: 19 jul., 2012

Aceito: 10 out., 2014

\section{AUTOR PARA CORRESPONDÊNCIA}

\section{Wilian de Oliveira Rocha}

Grupo de Produção Acadêmica de Ciências Agrárias, Biológicas e Engenharias, Curso de Engenharia Ambiental, Centro Universitário de Várzea Grande - UNIVAG, CEP 78118-900, Várzea Grande, MT, Brasil

e-mail: wilianroch@hotmail.com

\section{REFERÊNCIAS}

Agosti D, Majer JD, Alonso LT, Schultz T, editores. Ants: Standard methods for measuring and monitoring biodiversity. Washington: Smithsonian Institution Press; 2000. [cited 2010 Aug 1]. Available from: http://antbase.org/databases/ publications_files/publication-20330.htm.

Aquino AM, Aguiar-Menezes EL, Queiroz JM. Recomendação para coleta de artrópodes terrestres por armadilhas de queda (pit-fall traps). Seropédica: Embrapa Agrobiologia; 2006. Circular Técnica n. 18.

Bestelmeyer BT, Schooley RL. The ants of the southern Sonoran desert, community structure and the role of trees. Biodiversity and Conservation 1999; 8(5): 643-657. http://dx.doi.org/10.1023/A:1008873406658.

Bihn JH, Verhaagh M, Brändle M, Brändl R. Do secondary forest act as refuges for old growth forest animals? Recovery of ant diversity in the Atlantic forest of Brazil. Biological Conservation 2008; 141(3): 733-743. http:// dx.doi.org/10.1016/j.biocon.2007.12.028.

Bolton B. Synopsis and classification of Formicidae. Memoirs of the American Entomological Institute 2003; 71: 1-370.

Cuezzo F. Revision del género Forelius (Hymenoptera: Formicidae: Dolichoderinae). Sociobiology 2000; 35(2A): 197-275.

Delabie JHC, Agosti D, Nascimento IC. Litter and communities of the Brazilian Atlantic rain forest region. In: Agosti D, Majer JD, Alonso LE, Schultz TR, editores. Measuring and monitoring biological diversity: standart methods for ground living ants. Washington: Smithsonian Institution; 2000.
Fowler HG. Ecologia nutricional de formigas. In: Panizzi AR, Parra JRP, editores. Ecologia nutricional de insetos e suas implicações no manejo de pragas. São Paulo: Manole; 1991.

Françoso RD, Corrêa RS. Riqueza de formigas e térmitas e sua contribuição para a recuperação de uma área minerada no Distrito Federal. In: Anais do VII Congresso de Ecologia do Brasil; 2007; Caxambú. Caxambú: Sociedade de Ecologia do Brasil; 2007.

Kremen C, Colwell RK, Erwin TL, Murphy DD, Noss RF, Sanjayan MA. Terrestrial arthropod assemblages: their use in conservation planning. Conservation Biology 1993; 7(4): 796-808. http://dx.doi.org/10.1046/j.15231739.1993.740796.x.

Majer JD. Ants: bio-indicators of minesite rehabilitation, land-use, and land conservation. Environmental Management 1983; 7(4): 375-383. http://dx.doi.org/10.1007/BF01866920.

Mato Grosso. Prefeitura Municipal de Poxoréu. Nossa cidade: caracterização do município [cited 2010 Feb 14]. Available from: http://www.poxoreu.mt.gov.br/ nossacidade/ caracterizacao.htm.

Moraes RCB, Haddad ML, Reyes AEL. Software para análise faunística - ANAFAU. In: Resumos do VIII Simpósio de Controle Biológico; 2003; São Pedro. Piracicaba: SEB; 2003. p. 195.

Oliveira MA. Diversidade da mirmecofauna e sucessão florestal na Amazônia - Acre, Brasil [tese]. Viçosa: Programa de Pós-Graduação em Entomologia, Universidade Federal de Viçosa; 2009.

Pacheco UP, Diniz JLM, Paniago GG, Santos GG, Lombardi MP. Observações e coleta de formigas da região de Jataí, Estado de Goiás (Hymenoptera, Formicidae). Jataí: Universidade Federal de Goiás; 2005. Relatório de Projeto de pesquisa.

Pereira MPS, Queiroz JM, Valcarcel R, Mayhé-Nunes AJ. Fauna de formigas como ferramenta para monitoramento de área de mineração reabilitada na Ilha da Madeira, Itaguaí, RJ. Ciência Florestal [Internet] 2007 [cited 2009 Sept 1];17(3) :197-204. Available from: http://redalyc. uaemex.mx/redalyc/pdf/534/53417302.pdf.

Ré TM. O uso de formigas como bioindicadores no monitoramento ambiental de revegetação de áreas mineradas [tese]. São Paulo: Escola Politécnica da Universidade de São Paulo; 2007.

Ribeiro JF, Walter BMT. Fitofisionomias do bioma cerrado. In: Sano SM, Almeida SP, editores. Cerrado: ambiente e flora. Planaltina: Embrapa Cerrados; 1998.

Rocha PS. Validação de bioindicadores de recuperação de áreas degradadas por mineração de bauxita em Poços de Caldas, MG [dissertação]. Rio Claro: Instituto de Geociências e Ciências Exatas, Universidade Estadual Paulista; 2007.

Rocha RA, Solferini VN. Número de rainhas e acasalamentos em Camponotus crassus (Mayr, 1862). In: Anais XVI 
Congresso Interno de Iniciação Científica; 2008; Campinas. Campinas: [s.n.]; 2008. [cited 2010 Jan. 20]. Available from: http://www.prp.unicamp.br/pibic/congressos/ xvicongresso/cdrom/FSCOMMAND/pdfN/341.pdf.

Rocha WO. O garimpo de diamantes como fator da degradação ambiental no Município de Poxoréu - MT. In: Sociedade Brasileira de Recuperação de Áreas Degradadas. Curitiba; 2008. [cited 2009 Aug 29]. Available from: http://www. sobrade.com.br/index.php?option=com_content\&task= category\&sectionid $=5 \& \mathrm{id}=16 \&$ Itemid $=44$.

Santos MS, Louzada JNC, Dias N, Zanetti R, Delabie JHC, Nascimento IC. Riqueza de formigas (Hymenoptera, Formicidade) da serapilheira em fragmentos de floresta semidecídua da Mata Atlântica na região do Alto do Rio Grande, MG, Brasil. Iheringia, Série Zoologia 2006; 96(1): 95-101. http://dx.doi.org/10.1590/S0073-47212006000100017.

Silveira Neto S, Nakano O, Barbin D, Villa Nova NA. Manual de ecologia dos insetos. Piracicaba: Ceres; 1976.

Silveira Neto S, Monteiro RC, Zucchi RA, Moraes RCB. Uso da análise faunística de insetos na avaliação do impacto ambiental. Scientia Agricola [Internet]. 1995; [cited 2009 Sept 2]; 52(1): 9-15. Available from: http:// www.scielo.br/pdf/sa/v52n1/02.pdf.

Valentim CL, Solar RRC, Schmidt FA, Ribas CR, Schoereder JHl. Formigas como bioindicadoras de impacto ambiental causado por arsênio. In: Biológico, XVIII Simpósio de Mirmecologia; 2007; São Paulo: [s. n.]; 2007. p. 297-300.

Valentim CL. Formigas como bioindicadoras de impactos ambientais e de reabilitação de áreas após atividades de mineração [dissertação]. Visçosa: Programa de PósGraduação em Entomologia, Universidade Federal de Viçosa; 2010.

Vasconcelos HL. Effects of forest disturbance on the structure of ground-foraging ant communities in central Amazonia. Biodiversity and Conservation 1999; 8(3): 407-418. http://dx.doi.org/10.1023/A:1008891710230.

Wink C, Guedes JVC, Fagundes CK, Rovedder AP. Insetos edáficos como indicadores da qualidade ambiental. Revista de Ciências Agroveterinárias [Internet]. 2005 [cited 2009 Sept 1];4(1): 60-71. Available from: http://rca.cav.udesc. br/rca_2005_1/wink.pdf. 